Cinémas

Revue d'études cinématographiques

Journal of Film Studies

\title{
Mémoire d'un achèvement. Approches de la fin dans les Histoire(s) du cinéma, de Jean-Luc Godard
}

\section{André Habib}

Volume 13, numéro 3, printemps 2003

Imaginaire de la fin

URI : https://id.erudit.org/iderudit/008705ar

DOI : https://doi.org/10.7202/008705ar

Aller au sommaire du numéro

Éditeur(s)

Cinémas

ISSN

1181-6945 (imprimé)

1705-6500 (numérique)

Découvrir la revue

Citer cet article

Habib, A. (2003). Mémoire d'un achèvement. Approches de la fin dans les Histoire(s) du cinéma, de Jean-Luc Godard. Cinémas, 13(3), 9-31.

https://doi.org/10.7202/008705ar
Résumé de l'article

Une des avenues les plus riches pour penser la fin au cinéma consiste à replacer sa question au plus près d'une pensée de la fin $d u$ cinéma. Cet article tentera d'interroger le sens de cette fin, comme lieu de sens, à partir et autour des Histoire(s) du cinéma (1987-1998), de Jean-Luc Godard. Il ne s’agira pas d'investir toute l'étendue de la question ni de proposer un historique de ces multiples « morts du cinéma », mais de tracer un certain nombre de pistes pour l'envisager, à partir de celles que les Histoire(s) proposent. D'abord, on se demandera si la fin du cinéma, pour Godard, n'est pas l'occasion d'un retour sur ce qui l'a perdu ou sur son origine (ses promesses, ses possibilités) et si, en un deuxième temps, le repiquage vidéastique ne s'offre pas comme une façon de réaliser, à rebours, le souvenir de certaines de ses potentialités, trahies ou méconnues. 


\section{Mémoire d'un achèvement. Approches de la fin dans les Histoire(s) du cinéma, de Jean-Luc Godard}

\section{André Habib}

\section{RÉSUMÉ}

Une des avenues les plus riches pour penser la fin au cinéma consiste à replacer sa question au plus près d'une pensée de la fin $d u$ cinéma. Cet article tentera d'interroger le sens de cette fin, comme lieu de sens, à partir et autour des Histoire(s) du cinéma (1987-1998), de Jean-Luc Godard. Il ne s'agira pas d'investir toute l'étendue de la question ni de proposer un historique de ces multiples "morts du cinéma", mais de tracer un certain nombre de pistes pour l'envisager, à partir de celles que les Histoire(s) proposent. D'abord, on se demandera si la fin du cinéma, pour Godard, n'est pas l'occasion d'un retour sur ce qui l'a perdu ou sur son origine (ses promesses, ses possibilités) et si, en un deuxième temps, le repiquage vidéastique ne s'offre pas comme une façon de réaliser, à rebours, le souvenir de certaines de ses potentialités, trahies ou méconnues.

\section{ABSTRACT}

One of the richest ways to think about the end in film is to place the question closer to a reflection regarding the end of film itself. This article attempts to question the meaning of this ending, as a locus of meaning, by taking Jean-Luc Godard's Histoire(s) du cinéma (19871998) as its point of departure. Far from assuming the entire breadth of the question or from proposing a historical analysis of the multiple "deaths of cinema," this 
article traces a select number of paths by which this end might be imagined from among those proposed by Histoire(s). These paths will be guided firstly by the question of knowing if the end of cinema for Godard is not also the opportunity of a return to its origins (its promises and possibilities) and to that which lost it on its way, and secondly, if the videomaker's rerecording does not offer, through its backward gaze against the grain, another way to realize the memory of a certain number of its betrayed or misunderstood potentialities.

Mais où est le péril,

Là croît aussi ce qui sauve.

Hölderlin, cité dans Heidegger 1995, p. 355.

Les choses prennent du sens lorsqu'elles finissent. C'est parce que c'est là que l'histoire commence.

Godard 2001, p. 12.

Jean-Luc Godard a été à l'avant-scène de toutes les crises du cinéma depuis 1960 (destructeur, créateur, professeur de diagnostics, annonciateur saturnien). Il serait même possible d'affirmer que tout le discours contemporain sur la fin du cinéma a été informé, en grande partie, par ses pronostics. Ceci explique aussi que la figure de la "mort du cinéma», dans son œuvre et ses prises de parole, se présente, comme l'a excellemment montré Michael Witt (1999, p. 331-346), sous une multiplicité de formulations, qu'elle soit d'emblée ouverte au paradoxe, à la contradiction, à la contestation. De l'iconoclaste débonnaire qui, à l'époque de Pierrot le Fou, «attendait la fin du cinéma avec optimisme" (Godard 1998b, p. 257) à son intervention crépusculaire dans Chambre 666 (1984) de Wenders («le cinéma va mourir, très jeune»), sans oublier les mélancoliques litanies dont il émaille ses films et ses entretiens depuis les années 1980, la disparition du cinéma est cet inexorable horizon: à la fois à venir, présent, déjà passé. En tout cas, sa génération de cinéastes, ceux qui ont fait la Nouvelle Vague, 
incarnerait, tel qu'on l'entend dans les Histoire(s) du cinéma, le dernier souffle du cinéma : "Notre seule erreur fut de croire que c'était un début " $\left(3 \mathrm{~b}^{1}\right)$, alors que, très clairement, pour Godard, la Nouvelle Vague en constitue la fin. Qu'est-ce qui autorise une telle affirmation?

Les cinéastes-critiques des Cahiers occupent, on l'a dit, une place privilégiée dans l'histoire du cinéma. Ils se sont trouvés témoins et porteurs d'une histoire, riche et complexe, mais encore animée et compréhensible, tout en longeant la frontière derrière laquelle allait se jouer, sous diverses formes, ce qu'on appela "la mort du cinéma» (Daney et Godard 1998, p. 161). Cette lucidité ou cette compréhension seront vécues avec plus ou moins d'intensité, de résignation ou d'ardeur par les différents cinéastes de cette génération de Français: Chabrol, Resnais, Marker, Rivette, Rohmer, Truffaut, Varda. Aucun, pourtant, n'aura autant fait cas de ce sombre pronostic que Godard, puisque la mort du cinéma, mais aussi sa sauvegarde, constitue un axe central, douloureux et poignant de son œuvre.

Cette prise de position serait tributaire, en un sens, de l'héritage dont il se veut la conscience et la mémoire vivante. Godard s'est toujours proclamé, tout en reconnaissant la responsabilité que cela encourait, "enfant du musée et de la libération». C'est dans cette double lignée, indissociable, qu'il découvre peu à peu sa propre généalogie. D'abord, dans la lignée du musée, c'est-à-dire de cet étrange musée imaginaire, parrainé par Langlois-MalrauxFaure. Son histoire (de l'art) du cinéma, celle à laquelle il travaille, bien entendu, dans les Histoire(s), reconnaît que toute image est historique en un sens précis. Elle nous provient toujours de l'épaisseur d'une médiation où se confondent des temps hétérogènes, des réseaux de sens, des pratiques médiatiques hétéronomes. C'est dans les galeries de ce musée, du cinéma et de l'art, qu'il a été exposé à la "fraternité des métaphores" (3b). C’est là que s'est tissé ce lien fraternel avec le passé, avec un passé qui pouvait, grâce au cinéma, se traduire au présent.

La seconde lignée de sa généalogie, qui lui a pris peut-être plus de temps à parcourir, consiste à penser le cinéma dans la foulée de la Seconde Guerre mondiale, de la bombe A, des camps d'extermination et de la Libération. Selon Daney (1996, 
p. 209), tout un pan du cinéma moderne, de Rossellini à Godard, d'Antonioni à Bergman, se définit à partir d'une posture devant l'irréversible, ou l'irréparable: Auschwitz, Hiroshima. À ces deux figures emblématiques de son discours s'est peu à peu esquissée une autre figure tout aussi marquante, celle du résistant (Moulin, Weil, De Gaulle). Nous pourrions alors dégager du discours godardien trois grandes figures tutélaires, par ailleurs reprises dans les Histoire(s) du cinéma: le Musée, les Camps, la Résistance. C'est sans doute la conscience de cette triade qui a engendré chez Godard, au cours de ses années d'apprentissage à la Cinémathèque, aux Cahiers, puis enfin, tout au long de sa pratique de cinéaste, un double discours où s'énonce, décliné dans tous les sens, à peu près ceci: nous venons après, et si nous voulons aller de l'avant, il nous faut remonter vers l'avant et résister à toutes les Occupations, là où elles se trouvent.

Il y a toujours eu, mais de façon plus accusée dans les années 1980-1990, un dédoublement de la problématique godardienne: 1) nommer la disparition d'un art, voire d'une fin de l'art occidental en général, qui aboutirait au cinéma-fin de la projection, c'est-à-dire, de la représentation; 2) se donner le droit d'effectuer, face à cet art toujours-en-train-de-disparaître, des remontées illicites vers une origine ou vers un pouvoir de résistance qu'il est encore capable de manifester. C'est ce que Dominique Païni a appelé un « ressourcement auratique» (1997, p. 167).

Il est en effet fort possible que nous rations le sens de cette fin — si fin, en effet, il y a —, si nous ne la pensons pas avec la conviction, certes désespérée, qu'elle puisse re-présenter un début, c'est-à-dire qu'elle puisse rendre à nouveau (au) présent une origine, un plan-originaire, une première image, arrachée, en quelque sorte, à la mort du cinéma. Toute l'histoire du cinéma godardienne, et le pouvoir de son invention, se jouerait dans un tel mouvement de déréliction et de reprise, de perte et de sauvetage. Or, ce qu'il y a à "ressusciter", apparaissant toujours abîmé, fragmenté, mutilé, en lambeaux brillants, ne peut pas être intégralement restauré, puisque ce qu'il y a à sauver s'ordonne autour de cette fin préalable, qui en constitue l'horizon ${ }^{2}$. Devant 
la saturation des images publicitaires et télévisuelles, l'hégémonie des médias de masse et une culture dévoyée de l'expression cinématographique qui empêchent toute "monstration", Godard persistera, durant toutes ces années, à croire en un pouvoir des images, douloureusement acquis. Ce n'est qu'en passant par là qu'on peut, paradoxalement, espérer retrouver le cinéma, tout en faisant l'économie de sa disparition. On remarquera que c'est sans doute par un même renversement que quantité de ses films des dernières années mettent en scène le tournage ou le projet d'un film irréalisé, qui abîme de l'intérieur, tout en le réalisant, le film en question (Passion, 1981, Forever Mozart, 1997, Éloge de l'amour, 2001).

Nous pourrions dire que sa recherche du cinéma s'est, dès les premiers films, mobilisée à partir d'un geste de réactualisation du passé dans un présent écartelé, ou encore, d'une plongée vers un possible du cinéma ou de l'art en général, qui aurait été oublié et qu'il s'agirait de réitérer. Et tout ceci nous conduirait à l'idée que, pour Godard, la fin n'est une clôture que dans la mesure où elle se constitue aussi en ouverture, qui découvre, par à-coups, une origine - sans cesse décentrée, démultipliée, qui appelle la recherche d'un avant (le langage, le nom, la chute, l'image, la parole), s'offre comme l'occasion d'un recommencement, toujours différé, qui maintiendrait, en même temps, le négatif en face: "L'image est bonheur, mais le néant séjourne auprès d'elle» (4b).

S’il s'est fait, avec les années, l'héritier et le passeur mélancolique d'une histoire du cinéma (singulière et plurielle) qui l'a traversé, c'est aussi en se nourrissant de la conviction, messianique, que l'art sauve le réel, et qu'il faut pour cela le sauver, avant qu'il ne disparaisse tout à fait. Et cette tâche lui revient. En ce sens, son œuvre, marquée de filiations et de paternités, apparaît à certains, à lui-même sans aucun doute, comme une des dernières tentatives, profondément solitaire, de poursuivre les intuitions fondamentales du cinéma — d'Epstein, d'Eisenstein, de Faure, de Langlois, de Bazin, de Daney —, dont les Histoire(s) seraient l'aboutissement, provisoire.

Les huit épisodes des Histoire(s) du cinéma, à la fois bilan, tombeau, testament, hommage aux morts, sont un essai de survivance, un dialogue, en forme de retour, avec une communauté 
de questions et de pratiques qui lui sont propres. Mais les Histoire(s) semblent aussi prendre acte d'autres tournants qui ont affecté l'exercice d'une pensée de l'histoire du cinéma. L'aventure des Histoire(s) débute par des conférences à Montréal, en 1978, l'année même du congrès de Brighton, d'une renaissance de l'intérêt pour le cinéma des premiers temps, puis dans les années où l'on a vu apparaître quantité "d'œuvres cinématographiques fondées sur une poétique de la ruine" (Aumont 1999, p. 169-170). Les Histoire(s) se trament au moment où, comme le rappelait Daney (1996, p. 214), «au-delà de sa crise, ou, qui sait, de sa "mort", le cinéma boucle une boucle, très loin en amont. Dialogue avec le muet». Aussi, au moment où l'on déniche et projette des copies de films inachevés, en ruine, on voit aussi s'implanter une "nouvelle cinéphilie ", grâce à l'incursion des magnétoscopes et des techniques vidéo, rendant concomitant "la découverte en état fragmentaire des films les plus anciens et le visionnement fragmenté des films" (Païni 2002, p. 34-40). La vidéo, nous verrons de quelle façon, marquerait, en la niant du même geste qu'elle la confirme, la fin d'un certain cinéma, tout en garantissant la possibilité de son historicisation. En somme, c'est à partir de quelques-uns de ces tournants de l'histoire du cinéma que cette amorce du retour, sur fond de disparition, a été pensée, et devient pensable, dans les Histoire(s). Mais il nous faut auparavant nous demander de quoi et où, dans l'histoire, serait mort le cinéma.

En effet, de quoi serait mort, ou de quoi mourra le cinéma? Dans les Histoire(s) et ailleurs, le cinéma est maintes fois appelé à personnifier l'enfance de l'art, c'est-à-dire, "sa priméité, cette immédiateté essentielle» (Aumont 1997, p. 65). Si le cinéma est mort, c'est qu'on l'aura empêché de devenir ce qu'il était: le "petit dernier» de l'histoire de l'art, le seul à avoir été immédiatement populaire, à conjuguer les vertus de la projection et de la révélation dans une forme qui pense. Doué d'une force poétique inouïe et d'une capacité à témoigner pour l'histoire, le cinéma aurait été étouffé par l'histoire du siècle, les industries de la mort et du sexe, les atrocités de la guerre, les grands médias de l'information, bref, par toute "cette épaisseur diffuse qui empêche que tout se montre»(4b). S’il est une fin du cinéma, 
elle apparaît tout d'abord, pour Godard, comme ce par quoi cet état d'enfance aura été perverti, tout ce qui l'aura empêché de "poursuivre son chemin vers l'enfance et dans l'enfance», lui qui avait "l'enfance pour patrie et origine» (Agamben 1989, p. 68). La mort du cinéma découlerait de sa faillite. Le cinéma était appelé à devenir quelque chose; s'il meurt — ou s'il est mort -, c'est faute d'avoir pu devenir ce qu'il était. Et son Waterloo, il le rencontre, selon Godard, à Auschwitz.

Tout au long des Histoire(s), c'est au cœur de cette profonde cassure qu’ont représentée les années 1939-1945 que se logerait le point nodal de cette faillite qui permet d'échelonner toutes les autres fins qui s'y manifestent: "La flamme s'éteint définitivement à Auschwitz", entend-on dans les Histoire(s) (3a). Mais comment comprendre le sens de cette phrase? Dotons-nous d'un exemple et tentons de voir quels sont les termes dans lesquels cette perte s'exprime. Un court segment de l'épisode $1 \mathrm{~b}$ nous servira de guide.

Ce segment se présente à la suite d'une série constituée autour de Zola et de la photographie. Plusieurs titres des romans de Zola ont défilé à l'écran: La Bête humaine, Nana, Au bonheur des dames, puis, pour terminer, J'accuse. C'est en jouant sur la double autorité de ce dernier titre que Godard va organiser une part de son ré-enchaînement. J'accuse, c'est évidemment le pamphlet de Zola, publié dans L'Aurore, en 1898, et dans lequel il prenait parti pour la révision du procès de Dreyfus. Mais J'accuse est aussi un film d'Abel Gance, tourné en 1919, à la suite de, et ayant pour cadre diégétique, la Première Guerre mondiale (une seconde version sera tournée en 1937). Dans le film de Gance, l'appel patriotique qui retentissait dans la bouche d'un poète, "Debout les morts!", se voulait un avertissement pour empêcher un autre conflit de cette ampleur. C'est à ce film - mais aussi à La Bête humaine - que Godard pense lorsqu'il poursuit, plus loin, avec un autre titre du même réalisateur, La Roue (1922), film dont les péripéties sont liées au transport ferroviaire ${ }^{3}$. Entre J'accuse et La Roue, Godard a tôt fait de tirer deux axes principaux, par l'entremise d'un montage fulgurant, entre L'Arrivée du train en gare (Lumière, 1895) et des images d'actualités présentant une déportée, émaciée, de la 
Seconde Guerre mondiale. Godard met en tension ici un des premiers mouvements captés par la cinématographie et la trace demeurée vive de sa plus grande défaite (nous verrons en quoi). Cette translation est opérée par le véhicule qui a symbolisé à la fois le triomphe de la pensée instrumentale et de la modernité au XIX siècle, et l'instrument privilégié pour mettre en œuvre la plus brutale des guerres d'extermination: le train.

L'express Lyon-Auschwitz que Godard nous fait prendre n'emprunte pas un trajet simple, puisqu'il se complique d'un réseau de sens supplémentaires. C'est un extrait de La Roue d'Abel Gance qui assure la médiation entre ces deux réalités: la naissance d'un art et la violence des camps. On voit une jeune fille accourir sur le quai d'une gare, cependant que, en surimpression, une locomotive se dirige vers elle, menace de la renverser. Comme s'il s'agissait d'un raccord dans l'axe du regard de la fille, le plan qui suit montre une déportée au visage décharné, debout dans un wagon, visible par une brèche entre deux lattes de bois. Aussitôt après, Godard fait alterner, par un clignotement de plus en plus rapide, L'Arrivée du train en gare avec une photographie des roues d'un wagon. Cette alternance des plans (gros plan des roues et plan moyen de l'arrivée du train) produit l'illusion d'une accélération foudroyante du train des frères Lumière.

La mise en garde du J'accuse, à la fois de Gance et de Zola, résonne encore, sans avoir à être évoquée directement: "Debout les morts!» Gance ne pouvait se douter, au moment où il faisait prononcer cette exhortation, au lendemain de la drôle de guerre, que le train suivant était déjà en route, et que l'horreur qu'il n'avait fait qu'entrevoir allait écraser tous les espoirs. Mais n'étaitce pas aussi la tâche du cinéma de prévenir un nouvel embrasement, de remettre en esprit l'antisémitisme qui gangrenait la société française? Le raccord faux entre le regard de la jeune fille dans la gare, qui apparaît dans ce montage, et le visage horrifique de la déportée, nous dit la faillite vraie de cet espoir, qui est aussi, suivant Godard, la faillite de l'espoir du cinéma: c'est là que s'est jouée son épreuve de vérité. C'est alors entre L'Origine du monde et un Monde perdu (2a) que le cinéma serait appelé à être pensé, et que s'élaborent les termes de sa responsabilité. 
On reconnaîtra ici l'idée que Godard défend, depuis les années 1980-1990, d'un rendez-vous manqué du cinéma avec l'Histoire et avec sa propre histoire. Ce que le cinéma rencontre à Auschwitz, ce serait un manquement à ses devoirs, auxquels le réel est venu l'arracher, le dérobant du coup de cette vie qui lui avait était promise et qu'il était voué à restituer. C'est cette faillite que résume Rancière (2001, p. 227) lorsqu'il décrit cette "faillite à l'égard du siècle, dépendante d'une faillite du cinéma à son propre égard. La première concerne l'incapacité du cinéma dans le désastre de 39-45 et tout particulièrement son incapacité à voir et à montrer les camps de la mort».

À quoi répond-elle, plus précisément, cette faillite? Pour Godard (1998d, p. 404), "cela est arrivé, à un moment où on n'a pas voulu voir le monde dans l'état où l'avaient mis les camps». Le cinéma a été mis hors circuit parce qu'il "devenait dangereux, non pas de raconter des histoires, mais de voir l'histoire" (1998d, p. 402). Le cinéma, au moment où il devait passer des intrigues, des petites histoires à la grande fable désenchantée de l'histoire, aurait rompu avec sa propre puissance d'historicité. Le drame du cinéma aura été de devoir témoigner pour l'histoire, mais dans une histoire qui a méconnu son potentiel de témoignage. Cette faillite serait d'autant plus lourde à encaisser que le cinéma avait déjà, de Chaplin à Murnau, de Renoir à Lubitsch, de Gance à Lang, annoncé les camps, bien que le constat ne peut être fait que rétroactivement. On aurait, au bout du compte, préféré la camelote des intrigues à la monstration de l'histoire qui s'exprimait dans ses fictions.

Ce que le cinéma découvre, par ailleurs, c'est un pouvoir de révélation, une "mission sacrée", qui ne lui est apparue, paradoxalement, qu'une fois niée. D'où la place que Godard accorde au cinéma d'actualités dans les Histoire(s), le seul qui, durant ces terribles années, aurait tenté d'accomplir, même maladroitement, sa vocation de présence: "Un simple rectangle de $35 \mathrm{~mm}$, même rayé à mort, sauve l'honneur de tout le réel» (1a). De la même façon, il aura suffit de Rome ville ouverte (1946), ("parce qu'il a été fait par des gens sans uniforme»), et du "Je lutte», prononcé par Elina Labourdette, la plus jeune des Dames du Bois de Boulogne (Bresson, 1943), pour que «l'Italie regagne les droits de 
se regarder en face» (3a), pour que le cinéma français reconnaisse son pouvoir de résistance - d'où l'importance persistante de Bresson dans les Histoire(s) et dans Éloge de l'amour.

Ce qui est en cause pour Godard, c'est l'écoute de l'histoire, la mise en jeu de l'homophonie: le cinéma-conteur n'était pas seulement appelé à raconter des histoires mais aussi à témoigner, même par ses fictions, pour l'histoire. Godard nous en propose, ailleurs, une fable, au milieu de l'épisode $4 \mathrm{~b}$ des Histoire(s). Il s'agit en fait d'un roman de Charles Ferdinand Ramuz, Les Signes parmi nous, narré à l'aide de quelques cartons, sur une musique d'Arvo Pärt, narration entrecoupée — on dirait illustrée — d'images et de sons :
Il y avait un roman de Ramuz/qui racontait qu'un jour/un colporteur arriva dans un village/au bord du Rhône/et qu'il devint ami avec tout le monde/parce qu'il savait raconter mille et une histoires./Et voilà qu'un orage éclate/et dure des jours et des jours et des jours./Alors le colporteur raconte/que c'est la fin du monde./ Mais le soleil revient enfin/et les habitants du village chassent le pauvre colporteur./ Ce colporteur,/ C’ÉTAIT LE CINÉMA (4b).

À cette fable énigmatique, que Godard projetait à une époque de tourner, s'intercalent des séquences fragmentées de films: les phares d'une voiture, tirée de la Nuit du carrefour (Renoir, 1932), deux moments de King Lear (Godard, 1986), Fernandel dévalant un coteau, une mer en furie, des pulsations d'explosions dans la nuit, un train de déportés, un cadavre ramassé, une courte séquence de la fin de Psycho (Hitchcock, 1960) où Bates se tient immobile dans la lumière d'une ampoule vacillante, un coup de feu de Rio Bravo (Hawks, 1958).

Que raconte Godard ici, sinon un carrefour de l'histoire au tournant duquel, selon lui, la lumière du cinéma s'éteint ("éteignez les phares", entend-on sur la bande-son)? Le cinéma aurait montré que les signes étaient parmi nous, et que le monde n'a pas voulu les voir. Ces signes sont ceux, annonciateurs, de la fin d'un monde qu'aurait tenté d'énoncer le cinéma-colporteur, et c'est cette fin du monde qui, par son détour, allait expulser le cinéma du commerce avec les hommes, le propulsant vers sa fin. 
Nous pourrions dire que, dans la personne du colporteur se trouve conjuguée une triple image du cinéma: l’image du conteur (celui qui a voyagé, qui raconte), du marchand de camelote ("des signes bon marché», qui raconte pour vendre) et du témoin martyr (qui dit un état du monde et, à la fin, en est chassé). C'est donc bien, aussi, une trajectoire allégorique du cinéma qui s'offre en trois temps: le cinéma, le premier art immédiatement populaire, obtient l'assentiment des masses par son habileté à raconter des histoires : "Le cinéma projetait, et les hommes ont vu que le monde était là, un monde presque sans histoire, mais un monde qui raconte» (1b). C'est au cours de ce grand orage de la guerre que le cinéma est appelé à rejoindre l'histoire, à témoigner, à montrer la fin du monde. Les hommes n'en ont pas voulu, d'autant que le beau temps finit par revenir et qu'on voudrait effacer les signes de l'orage. Dès lors, le cinéma viendrait toujours après cet orage, qui l'aura écarté de sa communauté. En faire l'histoire consiste à relire toute son histoire à partir de cette perte, de cette expulsion: "L'histoire, sombre fidélité pour les choses tombées" (Péguy, Clio, cité dans l'épisode 4b).

Le raisonnement, forcément ambigu, est le suivant: s'il nous faut racheter le cinéma, c'est en partant de cette faillite, afin de tenter de redonner au cinéma la place qui lui revient, justement parce qu'il l'a perdue, dans l'histoire. Ce double mouvement, contradictoire mais fécond, de "devenir avant» ou de "retour en avant", est compris dans cette reconnaissance. On ne peut "faire l'histoire que de ce qui s'achève» (4b). A contrario, la fin permet de raconter l'histoire et, par le fait même, de résister à cette fin, en tentant de restaurer les possibilités que cette fin a dévoilées, en relisant et reliant les "mèches effilochées" de ce qui en elle survit, afin de montrer de quelle puissance de vie elles peuvent encore témoigner. Mais cela suppose, Rancière (2001, p. 228-229) l'a fort bien démontré, une dé-formation du sens de ces images et de ces sons, replacés dans le jeu vertigineux des métaphores et des homonymies qui révèlent un autre sens, qu'elles auraient elles-mêmes méconnu. La chasse aux lapins de La Règle du jeu (Renoir, 1939) comme préfiguration du génocide, les Teutons d'Alexandre Nevski (Eisenstein, 1938) en 
soldats de la Wehrmacht, le Sans avenir des Lumière transformé en «Sang à venir» de la Seconde Guerre mondiale.

L'imaginaire de la fin, chez Godard, se comprend à partir de la faillite d'un possible qui, à la fois, révèle ce possible et demande à le racheter. L'invention du parlant, l'invasion de la télévision, la réification des corps, le commerce des intrigues hollywoodiennes et l'hégémonie américaine scandent tous la fin de quelque chose qui appartenait à un domaine de l'art, unique, que le cinéma était appelé à conquérir et qui s'est vu détourné, renversé. À ces détournements de vocation se lient toutes les autres atrocités, que Godard met en série (et qui entrent en série avec Auschwitz): Hanoi, Sarajevo, Srebrenica, Gaza. Tous ces événements, ces lieux, ces dates deviennent, dans les Histoire(s) et ailleurs dans son ouvre (L'Origine du XXI siècle, 2000 ; Les enfants jouent à la Russie, 1993), les signes d'une catastrophe unique, permanente (comme le dit Duras dans Sauve qui peut (la vie), [1979]: "C'est la fin du monde tout le temps.»). Toutes ces horreurs du siècle sont aussi, pour reprendre le titre de l'épisode 4b, Les Signes parmi nous, ce que le cinéma nous permet d'imaginer, c'est-à-dire, pour Godard, de re-monter.

On comprend mieux l'analogie, déjà signalée, entre la posture de Godard et celle de l'Ange de l'histoire de Benjamin (Bergala 1999, p. 221-222), propulsé vers l'avant, mais les yeux tournés vers le passé, qui lui apparaît tel un «monceau de ruines [qui] devant lui s'élève jusqu'au ciel»: "Là où nous apparaît une chaîne d'événements, il ne voit, lui, qu'une seule et unique catastrophe, qui sans cesse amoncelle ruines sur ruines et les précipite à ses pieds. "Son vœu, désespéré, est «de réveiller les morts et de rassembler ce qui a été démembré»(Benjamin 2000a, p. 434). C'est d'un tel vou - rappelons-nous la formule de Gance que sont pétries les Histoire(s).

La dialectique de la catastrophe et du progrès à l'œuvre dans la figure de l'Ange, qui soutient les thèses sur l'histoire de Benjamin, Didi-Huberman (2000, p. 126-127) a récemment montré qu'elle se fondait sur un montage, ou plutôt sur un «mouvement suspendu qui figure à la fois, qui démonte et qui monte [...] la continuité d'un geste et son irrévocable interruption ". Ce double régime — qui consiste à démonter et 
remonter, relancer et interrompre - articule tout l'édifice, en ruine, des Histoire(s). Si Godard fragmente les récits, disloque l'ordre placide des chronologies et la succession des événements, c'est pour faire sourdre, par télescopages, ces arrêts et ces reprises d'histoire: il re-monte le passé, par fulgurance, en le faisant surgir comme image, une image entendue comme "phénomène originaire de présentation de l'histoire " (Didi-Huberman 2000, p. 124). Et c'est précisément à partir d'un travail de montage (historique, métaphorique, poétique) que Godard entend réaliser le cinéma, c'est-à-dire faire avec des films faits les films qui ne se sont pas faits (les dé-faire), qui serait la seule façon de tenter de racheter le souvenir d'une promesse. Cette pensée, "même sa propre impossibilité, elle doit la comprendre par amour du possible» (Adorno 1991, p. 230).

Dans le segment de l'épisode $1 \mathrm{~b}$ que nous signalions plus tôt, il est clair que c'est par la pratique du montage que le cinéma est capable de montrer, c'est-à-dire de témoigner, par relations interposées. Que nous dit Godard? La vocation du cinéma de nous faire accéder au réel s'éteint dans les fourgons en partance pour Auschwitz. Or, par l'emploi du montage et des plans d'actualités, il démontre, en même temps et à l'inverse, sa croyance dans le pouvoir du cinéma et dans la possibilité renouvelée de la mise en ouvre de sa puissance. C'est en ce sens que le montage témoigne du pouvoir de sens de l'image, capable de révéler à la fois la brutalité et la beauté, et dans un même mouvement, d'obtenir notre plus grand assentiment: "Le cinéma, comme le christianisme, ne se fonde pas sur une vérité historique. Il nous donne un récit, une histoire, et nous dit maintenant: crois " (1b).

Quelle est alors l'intelligence de l'histoire qui s'y manifeste? Nous pouvons dire, avec Agamben (1998, p. 68), qu'il s'agit d'une histoire messianique: une "histoire du Salut — il faut sauver quelque chose» - et une "histoire dernière", une "histoire eschatologique, où quelque chose doit être accompli, jugé, doit se passer ici, mais dans un autre temps, doit donc se soustraire à la chronologie, sans sortir dans un ailleurs». À la fois archéologue et chiffonnier, Godard s'est peut-être soustrait à un sens de l'histoire, et s'inscrit désormais dans l'histoire d'un sens 
historique des images, suivant un "esprit des formes", un Nachleben, une image-survivance, anachronique et intempestive, qui décloisonne le temps de l'histoire positive. Elle rejoindrait cette autre histoire qui, selon Jean-Luc Nancy (1993, p. 44),

\begin{abstract}
est en jeu, et qui nous fera relire toute notre histoire. Non plus l'histoire directionnelle et signifiante, d'un sens qui se déroule et se dégage, mais une histoire intermittente, stochastique et réticulée, traversée de pulsations plutôt que de flux. Non plus le sens de l'histoire, mais une histoire du sens - et pourtant, et en même temps, la relance d'une infinie libération.
\end{abstract}

Si le cinéma est, en quelque sorte, la mémoire de l'histoire du siècle (de toutes ses histoires), sa transmission ne peut être garantie qu'en passant par la mémoire. C'est dans cette idée qu'on retrouverait un sens de la communauté chez Godard, qui s'est substitué, en s'amplifiant et en s'adjoignant une cohorte de fantômes, à la bande des Cahiers: "La communauté est celle des morts, mais à condition que des vivants les fassent passer dans leur corps, en mémoire" (Aumont 1999, p. 220). De ce point de vue, nous pourrions dire que les Histoire(s) sont un essai de mémoire (mémoire de l'histoire, histoire d'une mémoire), qui essaie, bégayant, de rendre compte d'une histoire qu'il a traversée, de toutes celles qui ont transité par cet art, qui se sont projetées en lui, mais aussi celles qu'il a permis de projeter. Godard apparaît, en ce sens, comme le conteur de Benjamin (2000, p. 130) qui, «au terme de son existence, [...] voit défiler intérieurement une série d'images - visions de sa propre personne, dans lesquelles, sans se rendre compte, il s'est luimême rencontré». C'est dans ce montage foudroyant que la mort, comme le pensait Pasolini, écrit le sens d'une vie. Et il en serait du cinéma, sur ce point, comme de Godard lui-même (les deux termes sont devenus interchangeables dans les Histoire(s)) qui, arrivant au terme de leurs vies, tentent de rassembler les monceaux éparpillés de leur mémoire.

Il serait aisé de démontrer que, tout au long de sa carrière de cinéaste, Godard n'a jamais cherché autre chose que le cinéma, qu'il n'a cessé de le trouver et de le perdre. S’il le retrouve avec les bandes-vidéo des Histoire(s), est-ce, comme l'avançait Païni 
(1997, p. 184), parce qu’il est déjà perdu en tant que cinéma, et que, pour cela, "il faudrait donc la vidéo, bain fluidifiant ou linceul funèbre selon certains, pour suturer l'histoire du cinéma, et faire de cette histoire le constat mélancolique de sa disparition : on ne retrouvera jamais plus ce qui manque "? Comment faire l'économie de ce manque? Est-il possible que ce soit à partir de la vidéo que Godard tente d'accomplir, performativement, tout ce qui a manqué au cinéma, ou à tout le moins d'en proposer un souvenir, d'en exposer la méthode, voire une morale?

La vidéo permet, Godard l'a vu très vite, d'explorer une méthode qui participe de l'idée du cinéma, tout en lui étant extérieure, dans le registre des techniques, imposant une autre tournure ${ }^{4}$. Tout nouveau support, en effet, indique un contenu de sens, dicte une approche, institue des possibilités qui euxmêmes articulent les échanges symboliques, politiques, économiques d'un moment de l'histoire. Si, donc, Godard a toujours eu l'ambition un peu démesurée de retrouver, de sauver tout le cinéma, et ce parce que justement il en va de sa vie - et sauve qui peut - , c'est en s'accommodant et en inventant de nouvelles modalités pour effectuer cette reprise. Il y aurait donc toujours un présent, technique et historique, à l'œuvre dans toute cette opération de sauvetage.

En 1962, deux ans après la sortie d'À bout de souffle, Godard (1998a, p. 218) affirmait:

Il y a déjà eu Bresson, il vient d'y avoir Hiroshima, un certain cinéma vient de se clore, il est peut-être fini, alors mettons le point final, montrons que tout est permis. Ce que je voulais c'était partir d'une histoire conventionnelle et refaire, mais différemment, tout le cinéma qui avait déjà été fait.

En terminant les Histoire(s), c'est comme si, programmatiquement, il exauçait son vœu de 1962. Le trait décisif, il me semble, entre les deux moments, consisterait dans ce passage du film-pellicule (analogique) au film-vidéo (électronique). Entre $\grave{A}$ bout de souffle, film emblème du présent de la Nouvelle Vague, celui qui a incarné pour plusieurs générations une coupure 
franche dans l'histoire du cinéma, et les Histoire(s) qui bouclent la boucle du passé, tout en inachevant, comme nous l'avons vu, une histoire qui se clôt, se constituent, au fond, deux modalités de la répétition et de la différence, tournées vers un même horizon: reprendre, mais différemment, toute l'histoire des films qui se sont faits, entre reprise (tournée vers l'avant) et ressouvenir (pointé vers le passé).

La posture du Godard de 1986 exprime, nous le disions, une prise en charge d'un moment de l'histoire. Si cette ambition, dans la fougue de la jeunesse, paraissait encore réalisable sur pellicule, dans le présent revendiqué haut et fort par cette génération de 1960 , elle ne l'est déjà plus quand il commence à monter ses Histoire(s), au moment où, pour lui, le cinéma dont il veut témoigner ne se conjugue peut-être plus qu'au passé (bien qu'il continue, jusqu'à aujourd'hui, à tourner sur pellicule).

Se confiant à Serge Daney, à la fin des années 1980, Godard affirme :

Mon but, donc, hélas, c'est comme ce petit poème de Brecht: "J'examine avec soin mon plan: il est irréalisable." Parce qu'il ne peut se faire qu'à la télé — qui réduit. Ou qui te projette, toi spectateur, mais alors tu perds connaissance, tu es rejeté. Alors qu'au cinéma, le spectateur était attiré. Mais on peut faire un souvenir de cette histoire projetable. C'est la seule, et c'est tout ce qu'on peut faire (Godard et Daney 1998, p. 161).

S’il s'agit, comme nous le disions, de racheter le cinéma de sa faillite devant les catastrophes du siècle, comment faire cette histoire, qui se projetait, avec un médium qui ne peut que diffuser, programmer, réduire? La vidéo pourrait-elle fournir un souvenir et préserver l'idée de cette projection, montrer le cinéma en procurant une image, en différé, de ses possibilités? En permettant de penser ce que le cinéma a créé (ou avait à créer), la vidéo, telle que Godard la pratique, ne réalise-t-elle pas alors les potentialités de ce cinéma, retrouvées en mémoire, réitérées dans le repiquage vidéastique?

Nous dirons que Godard trouve dans la vidéo le moyen de représenter les modalités des impressions que le cinéma, une fois, a laissées dans sa mémoire. Elle ne supplée aux "défaillances de 
mémoire", comme les reproductions d'œuvres pour Malraux, qu'en autant qu'elle doive passer par cette défaillance ${ }^{5}$. Devant ses rangées de cassettes, comme devant ses rayons de bibliothèque, Godard cherche non pas un objet, mais le souvenir d'un objet déjà vu, retrouvé parfois au hasard, sur son écranmémoire. C'est au contact de ces images que d'autres surgissent, qui demandent à être exhumées de leur oubli, et restaurées dans le présent de nouvelles séries associatives, plus facilement accessibles que sur support pellicule. Car c'est bien en tant que souvenirs de cinéma que les Histoire(s) se réalisent. Si chaque médium se souvient de celui qui l'a précédé (qui lui sert de contenu, dirait McLuhan), la vidéo permet un retour non seulement sur le cinéma, mais sur l'ensemble des arts, auxquels le cinéma participe, avec lequel il peut entrer en relation. La vidéo permettrait alors à Godard de poursuivre ce que, tout au long de sa carrière, il a tenté de mettre en scène, c'est-à-dire les raccords anachroniques et troublants entre une peinture et un roman, entre un film et une anecdote historique, entre une musique et une leçon politique, en d'autres mots, ce qu'il est convenu d'appeler «le montage", dont les Histoire(s) serait le grand ouvre.

Le montage représente ce que le cinéma a eu en propre, ce qu'il a toujours cherché, sans le trouver. «Le montage fait la spécificité du cinéma, et sa différence par rapport à la peinture ou le roman. [...] Son originalité, comme une plante qui n'est jamais vraiment sortie de terre, c'est le montage " (Godard 1998c, p. 242). C'est sur le montage que s'érige tout l'édifice conceptuel et programmatique des Histoire(s), précisément parce qu'il représente ce que seul le cinéma avait permis de mettre en lumière.

La vidéo est alors, eu égard au montage, réflexive et performative, renvoyant à ce que le cinéma a tenté de trouver, et parvenant à le réaliser à partir d'images et de sons de cinéma, d'actualités, de textes littéraires, etc. Le montage proposé dans les Histoire(s) se fonde, comme Agamben l'a fort bien vu, sur la répétition et l'arrêt. La réactualisation des images et des sons et la puissance de suspension de l'arrêt sont deux manières de détourner ou de "contrarier", dirait Rancière, la fable cinématographique, son flux narratif, afin de raconter l'histoire de leur possible, c'est-à-dire de les déloger des histoires singulières, pour 
les faire participer à une histoire du sens, des gestes, des formes et de l'expression. C'est par ce détournement que la vidéo «rendrait possible» le montage cinématographique, en montrant justement toute les possibilités de sens, d'affects, d'histoire que le montage permet de révéler. Le support ne disparaît pas dans la représentation (Godard ne cesse de montrer ces outils de travail), mais se montre, précisément, comme médium de montage. Et ce montage est, pour Godard, inséparable d'un travail de mémoire et d'histoire, qui en tresse les mèches disparates. C'est, en somme, parce que la mémoire remonte constamment, avec l'aide de l'imagination, ce qui s'est déposé en elle, qu'on accéderait à une image de l'histoire. Toute l'entreprise des Histoire(s) consisterait à radicaliser les possibilités de la vidéo, la répétition et l'arrêt - les deux transcendantaux du cinéma, selon Agamben (1998, p. 69) - en tant qu'elles révèlent les conditions de possibilité du cinéma, pour en faire des analogoi de la réminescence. Ces films resurgissent, alors, dans l'écart qui se creuse entre leur répétition et la différence qui les affectent, et c'est dans cet écart que se joue tout le travail de la mémoire (sur l'oubli).

La répétition et la réminiscence, comme formes de réitération, ne peuvent pas retrouver l'identique, le même. Plutôt, elles effectuent à nouveau la possibilité de ce qui a été, en faisant de "l'inaccompli un accompli, et de l'accompli un inaccompli" (Agamben 1998, p. 70). Si le passé est inachevé, c'est qu'il apparaît, pour Godard, avant tout comme phénomène de mémoire («l'avenir éclate parmi les souvenirs», 3b). La répétition des images et des sons est dès lors inséparable d'une forme d'anamnèse, où le passé remonte, non pas tel qu'il a été vécu, mais tel qu'il s'actualise dans une triple temporalité, dans ce triple présent dont parle saint Augustin (présent du passé, présent du présent, présent du futur). C'est ainsi qu'on dira que les survivances du passé surgissent au présent en se projetant sur l'oubli qu'elles ont traversé. C'est de cette forme de projection que les Histoire(s) du cinéma tenteront de faire un souvenir.

Si le cinéma est proverbialement «l'art du présent ", de quelle temporalité, à la lumière de ce que nous disions, est saisie la vidéo, du moins telle que la pratique Godard? Le seul présent 
de la vidéo est celui des télescopages, des ralentis, des manipulations qu'elle permet et engendre: un présent qui déplie et déploie le temps. Le présent de la vidéo n'est plus celui du tournage, c'est celui de la collision, de la rencontre entre des temps passés, dans un présent qui est peut-être celui de Godard se retournant, comme Orphée ou l'Ange de Klee, sur ce passé qui revient au moment où il a reconnu, dans un éclair, la possibilité d'un montage entre deux images, entre une image et un son. Ce présent est aussi, pour nous, le moment fulgurant où ces images se torsadent, tourbillonnent, fragilisées, sous nos yeux. Par ailleurs, si le film-pellicule maintenait, jusque-là, son intégrité physique, temporelle, la vidéo, elle, transporte, arrête, rembobine, avance, ralentit, se prête à la tactilité d'un montage presque sans matière, fantomatique. Mais c'est en cela qu'elle impose une autre forme de présence à ces images (présence d'une absence, qui pense en cette absence). La vidéo permet de montrer directement un processus à l'œuvre au moment où il s'effectue: "Seeing (with video) is thinking (live, with the image)" (Dubois 1992, p. 73).

La spécificité des Histoire(s), par rapport à certains films-essais des années 1980, c’est que ce travail s'opère presque strictement à partir d'images extraites du passé. Comme l'écrivait Agamben (1998, p. 69): "On n'a plus besoin de tourner, on ne fait que répéter et arrêter.» Et il ajoute: "C'est là une nouvelle forme épochale par rapport à l'histoire du cinéma. [...] On fait du cinéma à partir des images du cinéma. » C'est qu'il s'agit d'une mémoire qui se rappelle et qui relit et écrit, en images et en sons, l'histoire de ses mouvements erratiques, de ses frictions tectoniques, de ses aveuglantes explosions. C'est ainsi une autre forme d'enregistrement, non plus du réel, mais d'un travail sur $d u$ réel, des séries de captures qui sont à leur tour repiquées et réenchaînées. La répétition, compulsive, qui se loge à toutes les étapes et à tous les niveaux des Histoire(s), n'est peut-être pas innocente: des copies de films que Godard emploie au repiquage, de la répétition des titres de films, de livres, prononcés à haute voix devant sa bibliothèque ou derrière sa machine à écrire, du martèlement et des legati de la Steenbeck aux réapparitions des titres des épisodes, d'un chapitre à l'autre, la 
répétition relance le travail de la mémoire, puisqu'elle donne lieu, à chaque nouvelle apparition, à une nouvelle série de signes qui surgissent, par associations quasi involontaires, télescopant des fragments d'images du passé, des lambeaux émouvants de durée.

La vidéo, on le sait, est un outil pour penser le cinéma et elle est directement liée à son institutionnalisation, du moins, telle qu'elle s'envisage aujourd'hui. C'est à partir de supports vidéo que se sont rédigés la plupart des ouvrages contemporains sur le cinéma, et tout un pan de sa théorisation en est clairement tributaire. Mais si la vidéo permet de penser, en le montrant, le cinéma (cogito ergo video), pour Godard, c'est avant tout parce qu'elle permet de le repenser (de le penser comme nouveau) et de réactualiser le possible de son sens. La vidéo permet de penser le cinéma en tant que "forme qui pense», mais, comme nous le faisions remarquer plus haut, qu'on a très vite empêchée de penser, de témoigner, de monter/montrer.

C'est peut-être ainsi qu'il nous faut poser, dans les Histoire(s), la question de la fin. La ruine du cinéma, en rejoignant la vidéo (le magnétoscope), cette «machine à fabriquer de la ruine, celle des films", le ruinerait à nouveau, mais "au profit d'une autre histoire, une histoire de l'art du film» (Païni 2002, p. 40). Et la tâche de cette histoire consisterait à en prélever les survivances, comme autant de "traces des dieux enfuis » que Godard se plaît à citer (1b). L'histoire de l'art qu'il pratique trouverait sans doute alors sa plus belle définition dans celle qu'en donne Nancy (1993, p. 208) :

Il y a une histoire de l'art, mais c'est l'histoire de cela qui ne cesse de faire irruption ou effraction dans l'histoire, de cela qui s'y donne toujours fini, toujours repris. [...] Mais pour autant qu'il est cet étrange refrain — anfractuosité, naufrage, brèche — il est l'art, le fragment, toujours à nouveau son propre fragment.

La vidéo serait une façon de faire, avec des images de cinéma, un cinéma qui ne s'est jamais fait, qui n'a jamais existé en tant que tel, en prenant alors au sens propre et figuré l'affirmation: "Le vrai cinéma, est celui qui ne peut se voir» (3b). Le travail de 
l'historien ne consiste-t-il pas en «une description précise de ce qui n'a jamais eu lieu» (3a)? La question est de faire voir ce qui ne peut être $v u$, c'est-à-dire de tresser une vision, à partir de relations. C'est alors que, déformés, méconnaissables, tous ces films re-montent comme s'ils étaient vus pour la première fois, dans l'épaisseur spectrale de leur histoire, dans un temps feuilleté entre les ligaments de la mémoire ${ }^{6}$. Et c'est par là que Godard entend redonner au cinéma son histoire, sur le seuil toujours reculé de son achèvement: "Un souvenir de ce qui n'a jamais eu lieu»(Agamben, cité dans Aumont 1999, p. 180), prenant la parole en cette absence même.

Ce tombeau pour le cinéma, hommage et gîte pour les morts et pour le siècle, a ses fondations solidement plongées dans l'urgence d'un recommencement, dans le devenir et le déclin. Godard se répète que "la seule chose qui survive à une époque telle quelle, c'est la forme d'art qu'elle s'est créée» (4b). Et le cinéma survivra, si l'enfance de son art lui survit. C'est l'espoir le plus fécond et le plus désespéré: espoir de voir ranimer une croyance dans le cinéma et dans la capacité du cinéma de nous restaurer une "croyance en ce monde-ci, tel qu'il est" (Deleuze 1985 , p. 225), et ce tant qu'il durera.

\section{Université de Montréal}

\section{NOTES}

1. Histoire(s) du cinéma est composée de 4 chapitres, chaque chapitre comprenant à son tour deux sous-chapitres (1a, 1b, 2a, 2b, 3a, 3b, 4a, 4b). Suivant l'usage, les références à cette œuvre seront indiquées en respectant cette division et apparaittront entre parenthèses dans le corps du texte.

2. S'il est question d'origine, ici, elle ne peut être pensée comme ce qui restaure l'ordre et la cohérence, dans la placidité d'un ordonnancement régulé. L’origine n'est pas une totalité du monde. Elle n'est pas plus une genèse fixe, un pli premier dans l'ordre chronologique de l'histoire. De la même façon, la fin n'est, pas plus, le terme ultime qui, une fois atteint, liquide toute possibilité. Origine et fin seraient des termes à être dépassés dans leur acception fixe, dans la mesure où nous les concevons comme des pôles dynamiques, qui se relaient et se relancent, s'informent, se suppléent et se télescopent. Ils se situent, sans s'annuler, l'une par rapport à l'autre.

3. Sisif le mécanicien recueille une jeune fille après un accident de train; il sera aveuglé par un jet de vapeur, etc.

4. Il suffit de constater avec quelle constance Godard, depuis Ici et ailleurs (19701975), les séries télé France/Tour/Détour/deux/enfants (1975-1976), et en particulier les

Mémoire d'un achèvement. Approches de la fin dans les Histoire(s) du cinéma, de Jean-Luc Godard 
œuvres vidéo des années 1980-1990, travaille la vidéo. À ce propos, lire l'admirable texte de Philippe Dubois (1992): "Video Thinks what Cinema Creates: Notes on Jean-Luc Godard's Work in Video and Television».

5. André Malraux (1996, p. 16) écrit sur les nouvelles possibilités historiques que procuraient les reproductions des ouvres: "Nous disposons de plus d'œuvres significatives, pour suppléer aux défaillances de notre mémoire, que n'en pourrait contenir le plus grand Musée. Car un Musée Imaginaire s'est ouvert, qui va pousser à l'extrême l'incomplète confrontation imposée par les vrais musées: répondant à ceuxci, les arts plastiques ont inventé leur imprimerie.»

6. «L'observation de l'artiste peut atteindre une profondeur presque mystique. Les objets éclairés perdent leurs noms: ombres et clartés forment des systèmes et des problèmes tout particuliers, qui ne relèvent d'aucune science, qui ne se rapportent à aucune pratique, mais qui reçoivent toute leur existence et leur valeur de certains accords singuliers entre l'âme, l'œil et la main de quelqu'un, né pour les surprendre en soi-même et se les produire" (Valéry, cité dans Benjamin 2000, p. 149).

\section{RÉFÉRENCES BIBLIOGRAPHIQUES}

Adorno 1991: Theodor W. Adorno, Minima moralia. Réflexions sur la vie mutilée [1951], Paris, Payot, 1991.

Agamben 1989: Giorgio Agamben, Enfance et histoire. Dépérissement de l'expérience et origine de l'histoire [1978], Paris, Payot, 1989.

Agamben 1998: Giorgio Agamben, "Le cinéma de Guy Debord», dans Image et mémoire, Paris, Hoëbeke, 1998, p. 65-76.

Aumont 1999: Jacques Aumont, Amnésies. Fictions du cinéma d'après Jean-Luc Godard, Paris, P.O.L., 1999.

Benjamin 2000: Walter Benjamin, «Le conteur: réflexions sur l'œuvre de Nicolas Leskov» [1936], dans Euvres III, Paris, Gallimard, 2000, p. 114-151.

Benjamin 2000a: Walter Benjamin, «Sur le concept d'histoire» [1942], dans Euvres III, Paris, Gallimard, 2000, p. 427-443.

Bergala 1999: Alain Bergala, Nul mieux que Godard, Paris, Cahiers du cinéma, 1999.

Daney 1996: Serge Daney, La Rampe [1982], Paris, Cahiers du cinéma, 1996.

Daney et Godard 1998: Serge Daney et Jean-Luc Godard, "Histoire(s) du cinéma: Godard fait des histoires. Entretien entre Jean-Luc Godard et Serge Daney» [1988], dans Alain Bergala (dir.), Jean-Luc Godard par Jean-Luc Godard. Tome 2, Paris, Cahiers du cinéma, 1998, p. 161-173.

Deleuze 1985: Gilles Deleuze, L'Image-temps, Paris, Minuit, 1985.

Didi-Huberman 2000 : Georges Didi-Huberman, Devant le temps. Histoire de l'art et anachronisme des images, Paris, Minuit, 2000.

Dubois 1992: Philippe Dubois, "Video Thinks what Cinema Creates: Notes on Jean-Luc Godard's Work in Video and Television ", dans Raymond Bellour et Mary Lea Bandy, Jean-Luc Godard: Son + Image 1974-1991, New York, Museum of Modern Art, 1992, p. 169-185.

Godard 1998: Jean-Luc Godard, Histoire(s) du cinéma, Paris, Gallimard/Gaumont, 1998.

Godard 1998a : Jean-Luc Godard, «Entretien» [1962], dans Alain Bergala (dir.), Jean-Luc Godard par Jean-Luc Godard. Tome 1, Paris, Cahiers du cinéma, 1998, p. 215-236. 
Godard 1998b: Jean-Luc Godard, "Questionnaire aux cinéastes français» [1965], dans Alain Bergala (dir.), Jean-Luc Godard par Jean-Luc Godard. Tome 1, Paris, Cahiers du cinéma, 1998, p. 257.

Godard 1998c: Jean-Luc Godard, "Le montage, la solitude, la liberté » [1991], dans Alain Bergala (dir.), Jean-Luc Godard par Jean-Luc Godard. Tome 2, Paris, Cahiers du cinéma, 1998, p. 242-251.

Godard 1998d: Jean-Luc Godard, "Histoire(s) du cinéma: à propos de cinéma et d'histoire" [1996], dans Alain Bergala (dir.), Jean-Luc Godard par Jean-Luc Godard. Tome 2, Paris, Cahiers du cinéma, 1998, p. 401-405.

Godard 2001 : Jean-Luc Godard, Éloge de l'amour, Paris, P.O.L., 2001.

Heidegger 1995: Martin Heidegger, "Pourquoi des poètes", dans Chemins qui ne mènent nulle part [1949], Paris, Gallimard, 1995, p. 323-385.

Malraux 1996: André Malraux, Le Musée imaginaire [1965], Paris, Gallimard, 1996.

Nancy 1993: Jean-Luc Nancy, Le Sens du monde, Paris, Galilée, 1993.

Païni 1997 : Dominique Païni, Le Cinéma, un art moderne, Paris, Cahiers du cinéma, 1997.

Païni 2002: Dominique Païni, Le Temps exposé. Le cinéma, de la salle au musée, Paris, Cahiers du cinéma, 2002.

Rancière 2001 : Jacques Rancière, La Fable cinématographique, Paris, Seuil, 2001.

Witt 1999: Michael Witt, "The Deaths of Cinema according to Godard", Screen, vol. 40, n $^{\circ} 3,1999$, p. 331-346. 